\title{
Tailored Magnetic and Electric States in 3d-Metal-Insulator Films: Characterization and Applications
}

\author{
J.A. FEDOTOVA* \\ NC PHEP, Belarusian State University, 220088 Minsk, Belarus
}

\begin{abstract}
Progress in designing of new low-cost magnetoelectronic planar devices requires new artificial films combining tuneable magnetic and electric properties. In this context, metal-insulator films are prospective for synthesis of materials with tailored physical properties that could be controlled with films composition and synthesis regimes. Present overview covers the summary of recent experimental results on complimentary and systematic study of macroscopic and local magnetic properties of films using vibrating sample magnetometer and Mössbauer spectroscopy with respect to phase composition and structural analysis at nanoscale by X-ray absorption spectroscopy in the extended X-ray absorption fine structure range, transmission electron microscopy and high-resolution transmission electron microscopy. Specific relationship between films structure and resulting magnetic properties (SP relaxation, core-shell exchange interaction, perpendicular magnetic anisotropy) is considered. Effects of magnetic and electric percolation in films are discussed in correlation with synthesis regimes (atmosphere of deposition, temperature of the substrate) and films composition. Physical mechanisms and models describing magnetic and electric properties of composite films are analyzed. Finally, technological approaches are proposed for tuning films properties towards their desired combination with respect to application in designing of sensors and planar (non-coil) inductive elements.
\end{abstract}

DOI: 10.12693 /APhysPolA.125.944

PACS: 73.40.Gk, 73.40.Rw, 73.63.Bd, 62.23.Pq, 68.37.Lp, 68.37.Og

\section{Introduction}

One of the modern trends in applied materials science is developing new artificial multifunctional materials and heterostructures that would be able to accomplish multiple performance objectives in a single material $[1,2]$. By definition these materials should satisfy the requirements of tunable magnetic and electric properties accompanied with microminiature scale and arbitrary shape, compatibility with silicon processing planar technology, reproducible and reliable processing, low cost and low power consumption. The area of applications for such materials is very wide covering communications, sensing, energy storage, visualization of magnetic fields, transformers, electrical componentry, etc. [3]. Obviously, nanocomposite films are the best candidate to combine incompatible, sometime alternative properties, and to satisfy the requirements of being multifunctional material. Indeed, magnetic metal (M)-insulator (I) granular nanocomposite films $\mathrm{M}_{x}-\mathrm{I}_{100-x}(x-$ contribution of metal in at.\%, $20 \leq x \leq 80)$ containing nanoparticles of $3 d$-metal nanoalloy $(3-10 \mathrm{~nm}$ in size) dispersed inside various insulating matrixes $\left(\mathrm{Al}_{2} \mathrm{O}_{3}, \mathrm{SiO}_{2}, \mathrm{CaF}_{2}\right.$, $\mathrm{ZrO}_{2}, \mathrm{PbZrTiO}_{3}$, etc.) represent extremely flexible and adaptive class of nanostructures offering unique combination of magnetic, electric and magnetoresistive properties that could be tuned towards concrete industrial application [3, 4]. Technologically, this could be quite easily achieved by variation of either films composition

\footnotetext{
*e-mail: julia@hep.by
}

(ratio of metallic $(x)$ and insulator $(100-x)$ fractions, material of matrix) or deposition regimes (sputtering atmosphere, temperature of substrate). As a result, M-I film nanoheterostructures sintered with ion-beam sputtering technique possess saturation magnetization $M_{\mathrm{S}}$ up to $1800 \mathrm{kA} / \mathrm{m}$, coercive force $H_{\mathrm{C}}$ less that $4 \mathrm{kA} / \mathrm{m}$ (at room temperature (RT)), magnetic permeability $\mu$ up to 200 at $50 \mathrm{MHz}$ and resistivity $\rho$ ranged between $10^{-2}$ and $10 \Omega \mathrm{m}[5,6]$. Such combination of properties makes M-I film particularly prospective for high-frequency applications $[6,7]$. Further to industrial application, such materials are also intrinsically interesting to investigate their specific magnetic and electric properties with respect to phase composition and structure at nanoscale. This overview will cover some aspects of structure and morphology of M-I granular films with variable chemical composition and deposition regimes in correlation with their resulting magnetic and electric properties.

\section{Experimental}

The films were deposited by dc ion sputtering, using $2 \mathrm{keV}$ argon ion gun, at $0.28 \mathrm{~nm} / \mathrm{s}$ rate, to a thickness $1-4 \mu \mathrm{m}$ onto $\mathrm{Al}$ and glass-ceramic substrates. The base pressure in the chamber was $2.6 \times 10^{-4} \mathrm{~Pa}$, the argon and oxygen pressures equal to $6.7 \times 10^{-2} \mathrm{~Pa}$ and $4.2 \times 10^{-3} \mathrm{~Pa}$, correspondingly. Sputtering targets were composed of $\mathrm{Fe}_{45} \mathrm{Co}_{45} \mathrm{Zr}_{10}$ alloy plates, covered with strips of insulator $\left(\mathrm{Al}_{2} \mathrm{O}_{3}, \mathrm{CaF}_{2}, \mathrm{PbZrTiO}_{3}\right)$ that were located on top. In this way, by changing the area coverage of the constituents, the metal/insulator compositional ratio in the deposited films could be varied [8]. Composition of the films, set in the range of $(\mathrm{FeCoZr})_{x}\left(\mathrm{Al}_{2} \mathrm{O}_{3}\right)_{100-x}$ 
(31 $\leq x \leq 64$, at. $\%$ ), was confirmed to be close to nominal, by energy dispersive X-ray spectroscopy (EDX) in a scanning electron microscope and by the Rutherford backscattering spectrometry (RBS) [4].

X-ray diffraction (XRD) patterns were collected with an Empyrean PANalytical diffractometer using a diffracted beam graphite monochromator and an X'Celerator linear detector $\left(\mathrm{Cu} K_{\alpha}\right.$ radiation) at the grazing incidence of 5 degree with respect to the sample surface with the detector scanning the $2 \theta$ space over the 10-120 degree range. The data obtained were analysed using the profile fitting program FullProf [9] based on the Rietveld method. Microstructural studies were done by transmission electron microscopy (TEM), using Philips EM400T microscope operated at $120 \mathrm{kV}$, and Philips CM200 operated at $200 \mathrm{kV}$ for high resolution TEM (HRTEM). Extended X-ray absorption fine structure (EXAFS) and X-ray absorption near-edge structure (XANES) measurements were performed at beam line ID26 of the European Synchrotron Radiation Facility using high energy resolution fluorescence detection (HERFD) [10]. The absorption profiles were detected at Fe and Co $K$-edges probing intensity of respective $K_{\alpha_{1}}$ fluorescence decay. Analysis of the EXAFS spectra was performed using Viper package [11].

Study of the surface topography and magnetic contrast was made using Solver 47-Pro atomic force microscope (AFM) working in AC magnetic force microscopy (MFM) mode, with a tip of cantilever covered with a $\mathrm{CoCr}$ of $40 \mathrm{~nm}$ thickness layer. The obtained results of AFM profilometry and AC MFM magnetic scanning were processed using Solver-Pro AFM software in order to improve picture quality and enhance contrast.

The ${ }^{57} \mathrm{Fe}$ transmission and conversion electron Mössbauer spectroscopy measurements were performed using a conventional constant acceleration type spectrometer with a $40 \mathrm{mCi}{ }^{57 m} \mathrm{Co}$ in $\mathrm{Rh}$ source at the liquid nitrogen and the room temperatures. The dc magnetization was measured in the temperature range from $2 \mathrm{~K}$ up to $400 \mathrm{~K}$ and in magnetic fields up to $90 \mathrm{kOe}$ using the vibrating sample magnetometer (VSM) option of the Quantum Design physical property measurement system (PPMS). During measurements magnetic field was applied in direction parallel to the thin film surface. The diamagnetic response of the substrate has been subtracted from the raw data. Four-probe resistance $(R)$ and magnetoresistance $(M R)$ measurements were performed with the current perpendicular to the applied magnetic fields up to $80 \mathrm{kOe}$ in temperature range from $10 \mathrm{~K}$ up to $273 \mathrm{~K}$ using the Cryogenics Ltd closed-cycle refrigerator system. Transport mechanisms in various samples were compared basing on the resistivity $\rho=R S / l$, where $S$ and $l$ are surface area and length of the sample. The $M R$ is defined as $M R=100 \% \times[R(H)-R(0)] / R(0)$, where $R(H)$ is the resistance at the applied field. Some limitation in the range of low-temperature measurements of $(\mathrm{FeCoZr})_{x}\left(\mathrm{Al}_{2} \mathrm{O}_{3}\right)_{100-x}$ films with small $x$ occurred because of very high starting electric resistance. Measure- ments of admittance $G(f, T)$ is performed in a cryostat with a special PC-based control system at the measuring temperatures $T$ from $80 \mathrm{~K}$ to $373 \mathrm{~K}$ by $5 \mathrm{~K}$ steps. A setup for the $G(f, T)$ measurements used either precision AC bridge HIOKI 3532 LCR HiTESTER (for the frequency range of $50 \mathrm{~Hz}-100 \mathrm{kHz}$ ) or precision Hewlett Packard LCR-meter HP 4284A (for measurements at the frequencies $100 \mathrm{kHz}-10 \mathrm{MHz}$ ).

\section{Structure of granular films}

Investigation of phase composition and structure of nanocomposite films is very complicated and ambitious task that definitely requires application of several complimentary techniques. TEM including high-resolution mode is very informative technique for starting verification of films structure as a whole as well as structure of nanogranules and matrix [12]. TEM and selected area electron diffraction (SAED) images in Figs. 1a and b show that crystalline metallic nanogranules appear as dark or differently shaded gray contrast (due to various crystallographic orientation with respect to the analyzing electron beam), while matrix exhibits a uniform light-gray contrast, thus confirming desired granular structure of studied films. More precisely, HRTEM images elucidate structure of nanogranules and allow experimental determination of interplanar spacing (Fig. 1c). Basing on SAED analysis of films together with fast Fourier transform diffraction analysis of HRTEM images one could identify the type of crystalline structure of granules, that usually corresponds either to bcc or fcc structure. It is seen from Fig. 1c that HRTEM image reveals the agglomeration of nanoparticles with growing metallic contribution $x$. HRTEM image is also helpful for preliminary study of matrix structure that could be either amorphous (for $\mathrm{FeCoZr}-\mathrm{Al}_{2} \mathrm{O}_{3}$ film) [12] or crystalline (for FeCoZr$\mathrm{CaF}_{2}$ film) [13].

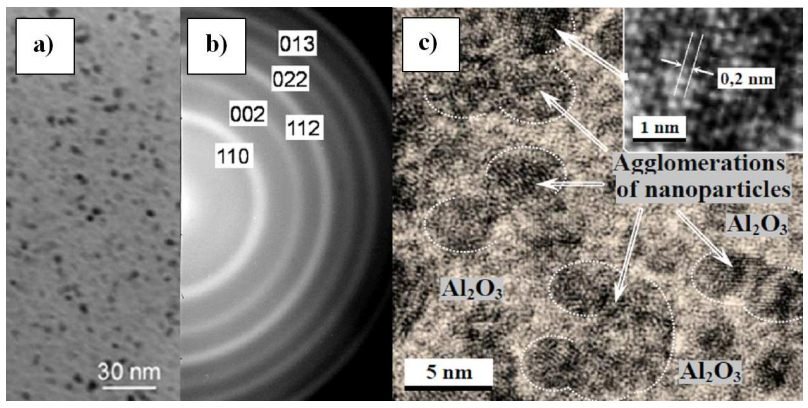

Fig. 1. Typical TEM [12] (a), SAED [12] (b), and HRTEM (c) images of $(\mathrm{FeCoZr})_{x}\left(\mathrm{Al}_{2} \mathrm{O}_{3}\right)_{100-x}$ films illustrating their granular structure and agglomeration of bcc FeCo-based nanogranules at $x>42$. The inset represent estimation of interatomic distance $d \approx 0.2 \mathrm{~nm}$ for $\alpha$-FeCo-based nanogranules.

Basing on TEM and HRTEM images three structurally different areas of films composition could be separated, 
particularly, $x<40$, where metallic nanoparticles are isolated inside matrix, $x \approx 50$, where granules start to agglomerate and $x>50$ characterized with pronounced agglomerations. It is noteworthy that composition around $x_{\mathrm{C}}=50$ that corresponds to so-called percolation threshold is an important characteristic of films and plays the key role in magnetic and electric properties to be considered in part 4 and 5 of present paper.

$\mathrm{X}$-ray diffraction analysis in glancing geometry is the most powerful tool to perform comprehensive characterization of metal-insulator granular films, i.e. identification of phase composition basing on profile fitting with the Rietveld method, calculation of lattice parameters $(a)$ and estimation of coherent scattering regions $\left(D^{\text {coh }}\right)$ with the Scherrer method. Basing on XRD patterns refinement nanogranules were identified as $\alpha$-FeCo-based nanoalloy with bcc cubic structure and $a=2.875-2.879 \AA[12,13]$.

Typical feature for nanogranules is the remarkable broadening of correspondent diffraction peaks much beyond the instrumental linewidth of the diffractometer. A decrease of the linewidth as well as gradual appearance of peaks at higher $2 \theta$ angles evidencing the long-range order in films is observed with increasing metallic concentration. To estimate the sizes of $D^{\text {coh }}$ the Scherrer method (in the form implemented in FullProf program [9]) was used to model the peak broadening (for the purely Lorentzian shape of the peak it reduces to $\Delta(2 \theta)_{L}=\left(\frac{2}{\pi}\right)\left(\frac{180}{\pi}\right) \frac{\lambda}{D \cos \theta}$, where $\Delta(2 \theta)_{L}$ denotes its full width at half maximum (FWHM), $\lambda=1.54056 \AA$ and $D$ corresponds to $D^{\text {coh }}$ ) above the instrumental width. Assuming that such broadening is solely due to size effects, the mean $D^{\text {coh }}$ of the crystalline grains was derived and comprised $\approx 1-4 \mathrm{~nm}[12,13]$.

In case of granular films analysed in present paper estimated grain sizes are usually in agreement with direct observations using TEM. It is noteworthy, however, that several authors note some inconsistency in application of the Scherrer formula for estimation of grain sizes in nanostructured materials [14]. This formula is derived assuming that diffraction peak is associated with a family of crystal planes, so that any deviation from translational symmetry (for example, structure imperfections or high proportion of atoms at granules surface) should make size estimations unreliable. Alternative approach is considered in [14], where the Fourier transform of diffraction patterns provides information about average distribution of interatomic distances in granules and makes estimate of particles size.

\section{Magnetic properties}

It is well established that magnetic properties of granular nanocomposites are fully defined with the size of metallic nanogranules (or their agglomerates) and, consequently, depend on metallic contribution $x[4,12,13]$. This is well illustrated in Fig. 2 that shows typical images of AFM and MFM for selected films with compositions around percolation, $x \approx 50$ and beyond percolation threshold, $x>50$ [4]. AFM images reveal that at studied magnification topography of the films are similar for all compositions (see Fig. 2a,c). At the same time, change of MFM images with increasing $x$ in films reflects variation of interparticle magnetic interaction. In particular, labyrinth-like MFM image at $x \approx 50$ is probably a signature of magnetic percolation (Fig. 2b). Above the percolation threshold, at $x>50$, stripe-like magnetic contrast (see Fig. 2d) confirms the formation of magnetically coupled net of the metallic granules $[4,12,15]$.

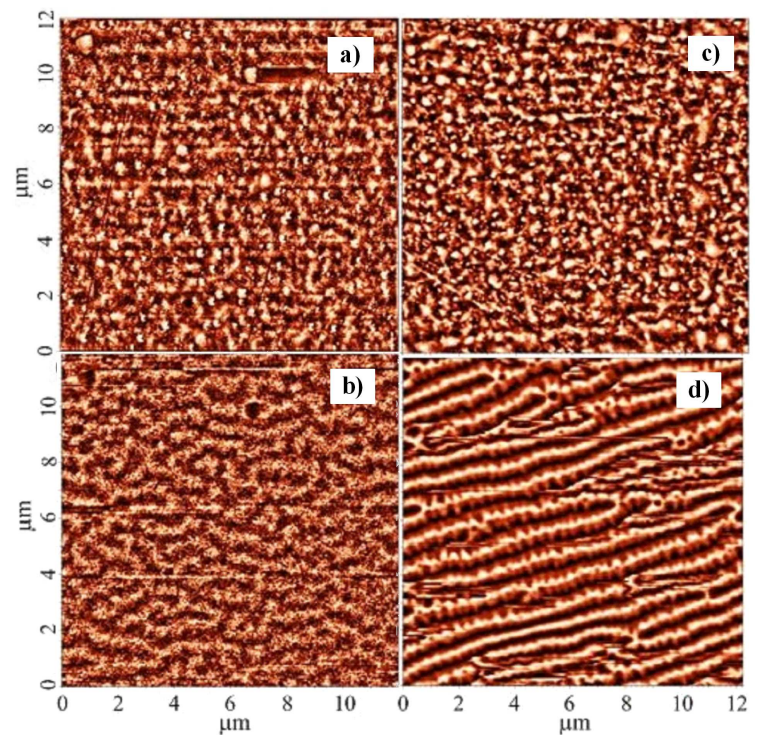

Fig. 2. Typical AFM (a, c) and MFM (b, d) images of $(\mathrm{FeCoZr})_{x}\left(\mathrm{Al}_{2} \mathrm{O}_{3}\right)_{100-x}$ films with $x \approx 50(\mathrm{a}, \mathrm{b})$ and $x>50(\mathrm{c}, \mathrm{d})[4]$.

Magnetic properties of selected granular films, namely magnetization as a function of temperature (in field-cooling FC and zero field-cooling ZFC modes) and external field, as well as correspondent Mössbauer spectra are illustrated in Fig. 3.

Brief consideration of presented complementary results provides comprehensive picture of magnetic state of films as a function of metallic contribution $x$. At $x<40$ the dependences of magnetization on temperature (in FC and ZFC modes) and external magnetic field as well as the Mössbauer spectrum recorded at RT are typical for the ensemble of superparamagnetic nanoparticles. Features of superparamagnetic relaxation are splitting of $M(T)$ curves measured in $\mathrm{FC}-\mathrm{ZFC}$ modes at blocking temperature $\left(T_{\mathrm{B}}\right)$ separating the areas of thermally fluctuating and blocked magnetic moments (see Fig. 3a), unsaturated $M(H)$ curve at RT even at high $H$ values, very small coercive force $H_{\mathrm{C}}$ (see Fig. $3 \mathrm{~d}$ ) and quadrupole doublet on the Mössbauer spectrum at RT (see Fig. $3 \mathrm{~g}$ ). Films with $x \approx 50$ that correspond to magnetic percolation are characterized with increased $T_{\mathrm{B}}$ (see Fig. 3b) and magnetically collapsed Mössbauer spectrum (Fig. 3h). This well correlates with labyrinth-like structure on MFM image (Fig. 2b) reflecting the onset of 


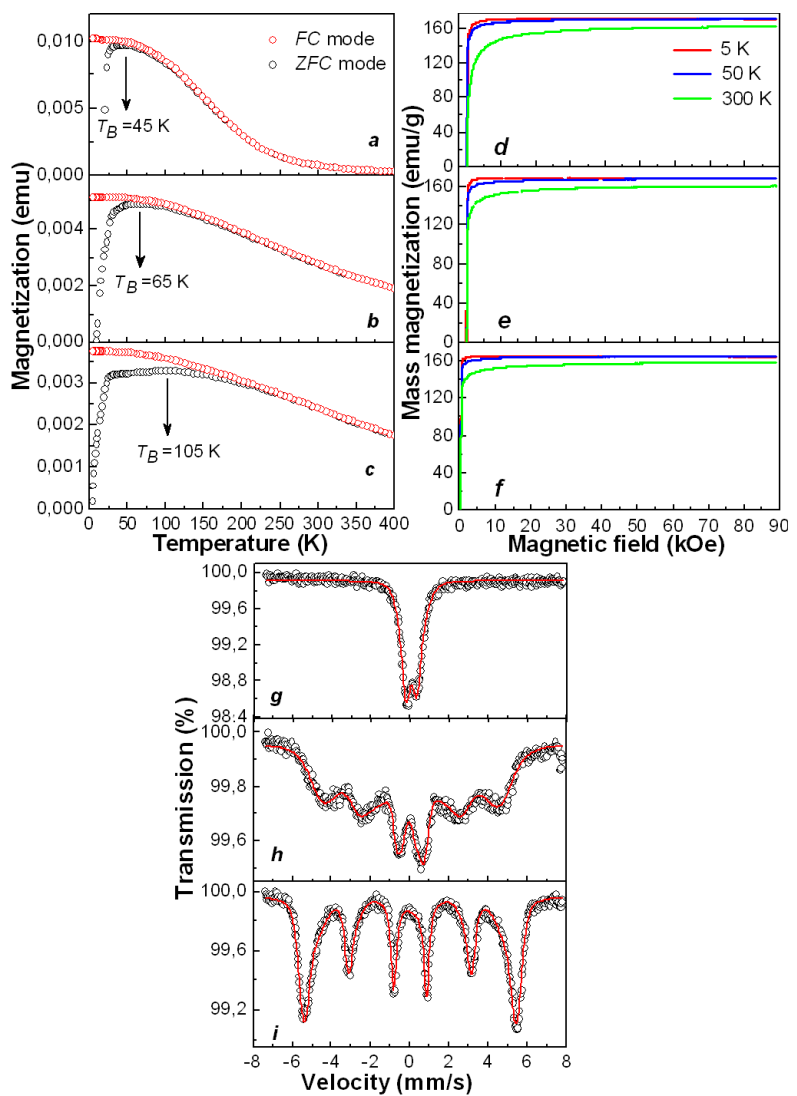

Fig. 3. Typical FC-ZFC curves (a)-(c), magnetization curves (d)-(f) and Mössbauer spectra (g)-(i) of $(\mathrm{FeCoZr})_{x}\left(\mathrm{Al}_{2} \mathrm{O}_{3}\right)_{100-x}$ films with $x<40$ (a, d, g), $x \approx 50(\mathrm{~b}, \mathrm{e}, \mathrm{h})$ and $x>50(\mathrm{c}, \mathrm{f}, \mathrm{i})[15,16]$.

ferromagnetic interaction between nanoparticles. Above magnetic percolation, at $x>50$, formation of magnetically ordered net of the metallic granules is proved with resolved magnetic sextet on the Mössbauer spectrum (see Fig. 3i) $[12,15,16]$.

Focusing more precisely on superparamagnetic state of nanoparticles, one should note that generally it means quasi-paramagnetic behaviour of materials containing very small magnetically ordered and weakly interacting particles [17]. Quasi-paramagnetic behaviour originates from thermal fluctuations of the magnetic moments of nanogranules around easy magnetisation axis with a finite probability that the moment will flip from one easy direction to another. The relaxation time $\tau$, which describes how rapidly the magnetic moment of a particle changes its alignment from one easy direction to another by thermal activation can be written in its simplest form, proposed by Néel and Hebd [18] as

$$
\tau=\tau_{0} \exp \left(\frac{K V}{k_{\mathrm{B}} T}\right)
$$

where $\tau_{0}$ is a characteristic time constant, $k_{\mathrm{B}}$ is the Boltzmann constant, $T$ is the temperature, $K$ is the uniaxial anisotropy constant, and $V$ is the volume of the par- ticle. Modification of this formula assuming characteristic time $\tau_{0} \approx 10 \mathrm{~s}$ and $10^{-8} \mathrm{~s}$ for VSM and Mössbauer spectroscopy, respectively, provides simple expressions for rough estimate of granules size: $25 k_{\mathrm{B}} T_{\mathrm{B}} \approx K V$ (for VSM) and $6.6 k_{\mathrm{B}} T_{\mathrm{B}} \approx K V$ (for the Mössbauer spectroscopy).

Superparamagnetic behavior of magnetically ordered nanogranules becomes apparent also on temperature evolution of the Mössbauer spectra from non-magnetic spectrum at RT through partially magnetically collapsed spectrum within $T_{\mathrm{B}}$ towards well magnetically resolved spectrum below $T_{\mathrm{B}}$. In case, when thermal energy $k_{\mathrm{B}} T$ is much stronger than magnetic anisotropy energy $\Delta E$, materials magnetization $M(H, T)$ could be approximated with Langevin function $L=\operatorname{coth}(a)-1 / a\left(a=\frac{\mu H}{k_{\mathrm{B}} T}\right)$ :

$$
M(H)=M_{\mathrm{S}} \int_{0}^{\infty}\left[L\left(\frac{M_{\mathrm{S}} V H}{k_{\mathrm{B}} T}\right)\right] f(V) \mathrm{d} V,
$$

where $f(V)=\frac{1}{\sqrt{2 \pi \sigma} V} \exp \left(-\frac{\ln ^{2}(V /\langle V\rangle)}{2 \sigma^{2}}\right)$. Such approximation for selected $\mathrm{FeCoZr}-\mathrm{Al}_{2} \mathrm{O}_{3}$ films provides realistic estimations of nanoparticles size $(\approx 5-11 \mathrm{~nm})[12,16]$.

\section{Mechanisms of charge transfer and magnetoresistance}

Experimental investigation of numerous $3 d$-based $\mathrm{M}-\mathrm{I}$ granular films have proved that similarly to their magnetic properties, mechanisms of charge transfer and magnetoresistance of films are related to the ratio between fractions of metal and insulator and thereby fall within percolation model [19]. According to this approach, at some definite concentration of metallic nanogranules - percolation threshold $x_{\mathrm{C}}$ - continuous currentconductive cluster between electric contacts on the composite sample is formed. Percolation transition within $x_{\mathrm{C}}$ results in the change of charge transfer mechanism from activational, variable range hopping ( $\mathrm{VRH}$ ), to metallic one and, consequently, the sign of temperature resistivity coefficient (TRC). Below the percolation threshold, in a dielectric regime granular films usually show negative TRC ( $\rho$ decreases with $T$ growth) and negative $M R$ effect. Above $x_{\mathrm{C}}$ the TRC becomes positive, while $M R$ effect falls up to near zero (or slightly positive) values. Experimental $\rho(T)$ curves for the films with $x<x_{\mathrm{C}}$ are approximated well either with Mott's $\left(\rho=\rho_{\infty} \exp \left(\left(\frac{T_{0 \mathrm{~m}}}{T}\right)^{0.25}\right)[20]\right)$ or the Shklovski-Efros VRH law $\left(\rho=\rho_{\infty} \exp \left(\left(\frac{T_{0 \text { se }}}{T}\right)^{0.5}\right)[21]\right)$, where $T_{0 \mathrm{~m}}$ and $T_{0 \text { se }}$ are characteristic temperatures of models.

M-I films are spintronic materials revealing spin-dependent tunneling (TMR) through insulating matrix between two neighboring metallic nanoparticles at $x<x_{\mathrm{C}}$. Typical experimental curves of $M R$ vs. $H$ (up to $80 \mathrm{kOe})$ at $\mathrm{RT}$ for selected $(\mathrm{FeCoZr})_{x}\left(\mathrm{Al}_{2} \mathrm{O}_{3}\right)_{100-x}$ granular films with $x=42$ and 47 are shown in Fig. 4a. Spin-dependent tunneling is proved with good correlation between $M R(H)$ and $\left(M / M_{\mathrm{S}}(H)\right)^{2}$ curves (see Fig. 4b). 

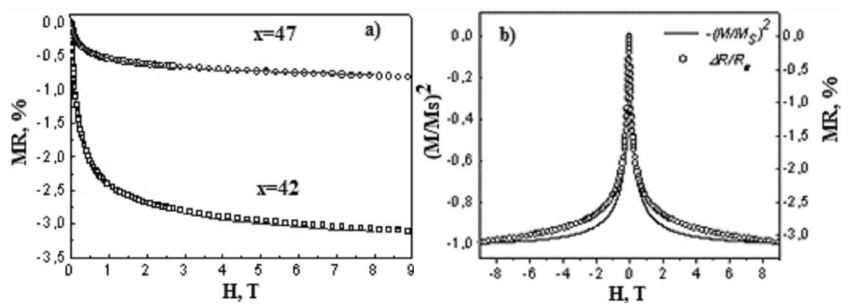

Fig. 4. Fitted $M R(H)$ curves at RT (a), correlation between $M R(H)$ and $\left(M / M_{\mathrm{S}}(H)\right)^{2}$ curves (b) for $(\mathrm{FeCoZr})_{x}\left(\mathrm{Al}_{2} \mathrm{O}_{3}\right)_{100-x}$ granular films.

$M R(H)$ curves could be well fitted within the frame of the Inoue-Maekawa model [22] with the following relationship:

$$
M R=-\frac{P^{2} m^{2}}{\left(1+P^{2} m^{2}\right)},
$$

where $P$ is tunnelling electron polarization, $m(H)=$ $\frac{M(H)}{M_{\mathrm{S}}}$, and $M_{\mathrm{S}}$ - saturation magnetization. Because of superparamagnetic status of metallic nanogranules at RT, $m(H)$ dependence could be approximated with the Langevin function $L(x)=\operatorname{coth}(x)-\frac{1}{x}$ applying the following expression:

$$
m(H)=\int_{0}^{\infty}\left[L\left(\frac{M_{\mathrm{S}}^{\mathrm{vol} V H}}{k_{\mathrm{B}} T}\right)\right] f(D) \mathrm{d} D,
$$

where $f(D)=\frac{1}{\sqrt{2 \pi \sigma} D} \exp \left(-\frac{\ln ^{2}(D\langle D\rangle)}{2 \sigma^{2}}\right)-\log$-normal size distribution of particle diameters $D,\langle D\rangle$ - mean particle diameter, $\sigma$ - the dispersion parameter, $M_{\mathrm{S}}^{\mathrm{vol}}$ - volume saturation magnetization of nanogranules (calculated from experimental $M(H)$ curves $[12,16]$ ).

Fitting of RT $M R(H)$ experimental curves with modified expression of the Inoue-Maekawa model performed with two tunable physical parameters - spin polarization $P$ and particle diameter $\langle D\rangle$ in general gives reasonable values $(P \approx 19$ and $\langle D\rangle \approx 3 \mathrm{~nm}[12])$ comparable with those extracted from the approximation of magnetization curves or direct TEM observations.

\section{Effects of sputtering ambient during deposition}

Variation of sputtering ambient, for example, sputtering in $\mathrm{Ar}+\mathrm{O}$ or $\mathrm{Ar}+\mathrm{N}$ gas mixtures proved to be very effective tool for tailoring of magnetic and electric properties of M-I granular films [4, 12, 13, 15, 16]. Particularly, synthesis of $\mathrm{FeCoZr}-\mathrm{SiO}_{2}, \mathrm{FeCoZr}-\mathrm{Al}_{2} \mathrm{O}_{3}$ and $\mathrm{FeCoZr}-$ $\mathrm{CaF}_{2}$ films in $\mathrm{Ar}+\mathrm{O}$ atmosphere resulted in the formation of nanogranules with "metallic core-oxide shell" structure (see Fig. 5) [12]. Formation of such a structure composed of crystalline metallic bcc $\alpha$-FeCo-based nanosized cores encapsulated in an amorphous oxide shell, all embedded in the amorphous $\mathrm{Al}_{2} \mathrm{O}_{3}$ matrix, is revealed with TEM and HRTEM (see Fig. 5).

X-ray absorption spectroscopy measurements in EXAFS range (see Fig. 6) exhibit selective oxidation of
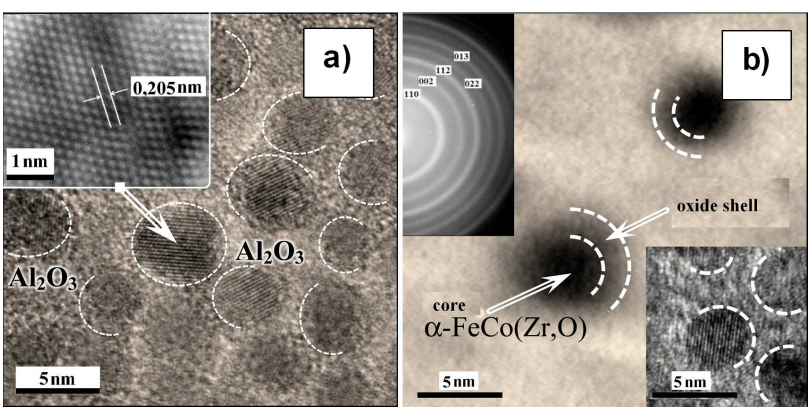

Fig. 5. HRTEM (a) and TEM (b) for $\mathrm{FeCo}-\mathrm{Al}_{2} \mathrm{O}_{3}$ film sintered in $\mathrm{Ar}+\mathrm{O}$ atmosphere [12].

nanoparticles that mean almost full oxidation of iron, while cobalt is found to be oxidized only partially [12].

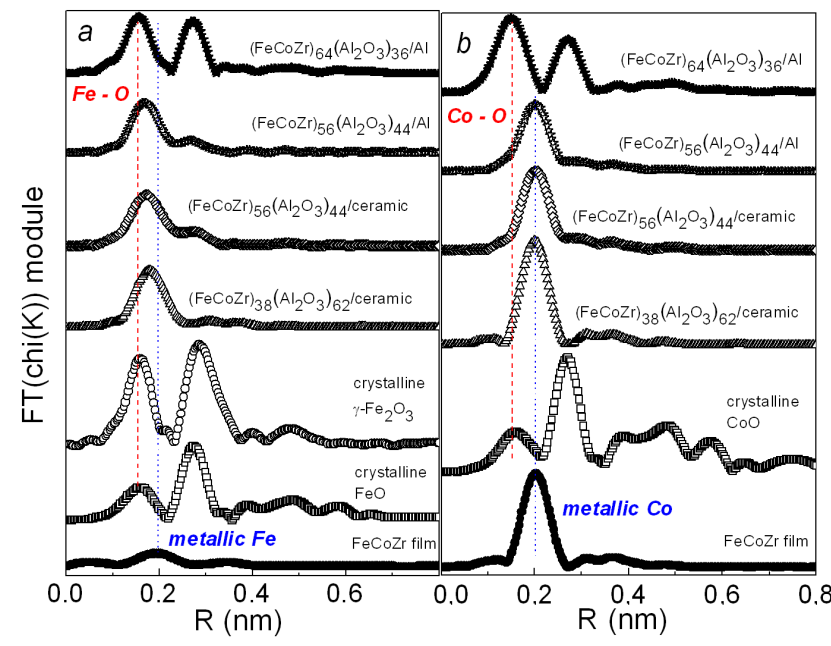

Fig. 6. Fourier transform of the EXAFS oscillations extracted from $\mathrm{Fe}$ (a) and $\mathrm{Co}$ (b) $K$-edge spectra. First maxima corresponding to oxide $(\mathrm{M}-\mathrm{O})$ and metal (metallic M) local environments are marked with dashed and dotted lines, respectively. Spectra are shifted vertically for clarity.

Such local atomic ordering in nanogranules correlates with the results of the Mössbauer spectroscopy, TEM and XRD, revealing that for $x \leq 56$ the metallic core constitutes bcc FeCo-based solid solution, possibly enriched with Co, while oxide shell mainly consists of mixed Fe-rich oxide [12].

Electric and magnetic properties of films containing nanoparticles with "core-shell" structure reflect the important role of oxide shells in preventing agglomeration, electric percolation and magnetic interparticle interactions $[4,12,13,15,16]$. Oxidized films reveal VRH (and/or tunneling) conductance in accordance with Mott's or Shklovski-Efros laws [20, 21] dominating for different $x$, and metallic conductive cluster network is not formed, thereby confirming vanishing of percolation transition. This should be related to the formation of oxide shells preventing coalescence of metallic nanogran- 
ules with increasing metallic contribution in films. For the same reason negative $M R$ of spin-dependent nature is observed for oxidized granular films in the whole range of $x$ values (see Fig. 7b) as compared to non-oxidized films (see Fig. 7a).

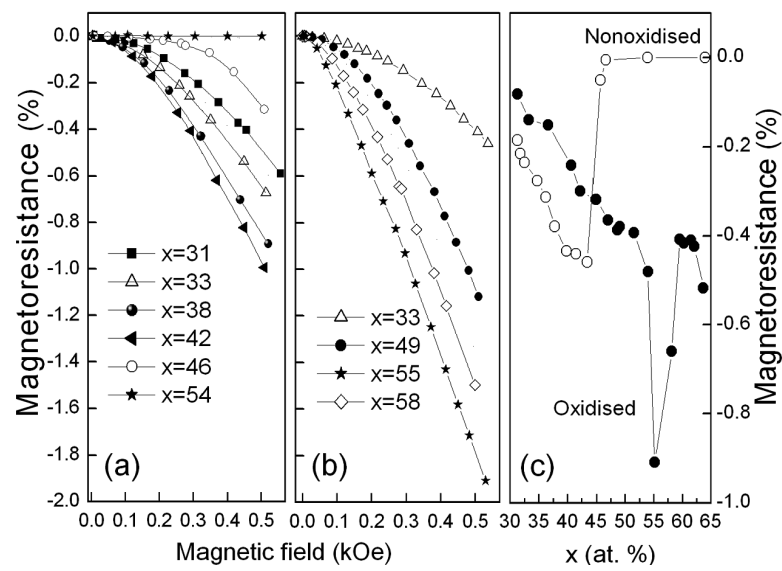

Fig. 7. $M R(H)$ curves for $\mathrm{FeCo}-\mathrm{Al}_{2} \mathrm{O}_{3}$ films sintered in $\mathrm{Ar}$ atmosphere (non-oxidized films) (a) and $\mathrm{Ar}+\mathrm{O}$ atmosphere (oxidized films) (b). $M R(x)$ for $\mathrm{FeCo}-\mathrm{Al}_{2} \mathrm{O}_{3}$ films at $H=0.3 \mathrm{kOe}(\mathrm{c})$ [12].

In addition, difference in the dependences of $M R$ vs. $x$ plotted at $H=0.3 \mathrm{kOe}$ for oxidized and non-oxidized composite films is well illustrated in Fig. 7c. It is seen that for non-oxidized films the crossover from negative to slightly positive $M R$ occurs within $x_{\mathrm{C}}$, while $M R$ for oxidised films remains negative up to $x=64$. Moreover, $M R$ for oxidised films within $x=55$ is enhanced up to twice with respect to the values characteristic for non-oxidized films. Such the enhancement of $M R$ in the films with the "core-shell" structure, shown in Fig. 7c, is assigned to the stabilization of the metallic cores sizes within optimal (single-domain) size owing to encapsulation inside oxide shells as well as to spin accumulation and spin filtering processes [12]. Oxidized films possessing $M_{\mathrm{S}}$ up to $695 \mathrm{kA} / \mathrm{m}, H_{\mathrm{C}}$ less than $0.02 \mathrm{kA} / \mathrm{m}$ and $\rho$ ranged between $4.6-540 \mathrm{~m} \Omega \mathrm{m}$ are very good high-frequency magnetically soft material.

The results of magnetometry on granular films $\mathrm{FeCoZr}-\mathrm{Al}_{2} \mathrm{O}_{3}$ synthesized in $\mathrm{Ar}+\mathrm{O}$ atmosphere support the formation of "core-shell" structures. It is consistent with non-saturating $M(H)$ curves at RT, as this means that partially oxidized FeCoZr granules remain in superparamagnetic state even for compositions above magnetic percolation at $x>50[15,16]$. Also the temperature dependence of coercive field $H_{\mathrm{C}}(T)$ was typical for the ensemble of non-interacting superparamagnetic magnetic nanoparticles revealing that for all analyzed compositions $H_{\mathrm{C}}$ values decreased with growing temperature $T$ and tends towards zero value at $T_{\mathrm{B}}$. According to the theory, temperature dependence of $H_{\mathrm{C}}$ for sample containing single-domain granules of equal size follows the relationship [23]:

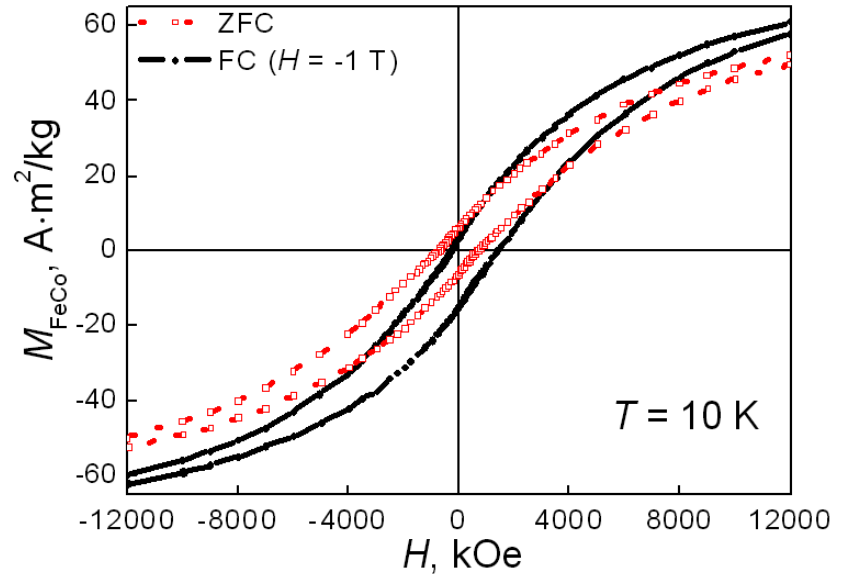

Fig. 8. Shift of $M(H)$ curve measured after FC at $T<T_{\mathrm{B}}$ for $\mathrm{FeCo}-\mathrm{CaF}_{2}$ film sintered in $\mathrm{Ar}+\mathrm{O}$ atmosphere relative to one measured in $\mathrm{ZFC}$ mode.

$$
H_{\mathrm{C}}=H_{\mathrm{C} 0}\left[1-\left(\frac{T}{T_{\mathrm{B}}}\right)^{1 / 2}\right],
$$

where $H_{\mathrm{C} 0}$ is $H_{\mathrm{C}}$ of the sample at $T=0 \mathrm{~K}$.

One of the typical evidence for "core-shell" structure of nanoparticles is the shift of magnetization curve measured after $\mathrm{FC}$ at $T<T_{\mathrm{B}}$ with respect to that measured after ZFC. The shift of hysteresis loop is often accompanied with an enhancement of $H_{\mathrm{C}}$ values observed after FC procedure. An example of such $M(H)$ curve shift for $\mathrm{FeCoZr}-\mathrm{CaF}_{2}$ is presented in Fig. 8. Such behavior is the signature of exchange AFM-FM coupling between ferromagnetic core and antiferromagnetic (or ferrimagnetic) oxide shell in case of its enough contribution [24].

\section{Effect of substrate temperature during films deposition}

It is recently proved that few systems like granular $\mathrm{Co}_{x} \mathrm{Ag}_{1-x}$ [25], $\mathrm{Co}-\mathrm{Zr}-\mathrm{O}$ [26] and $\mathrm{Co}-\mathrm{Al}_{2} \mathrm{O}_{3}$ films [27], $\mathrm{Co}-\mathrm{rich} \mathrm{Co}-\mathrm{Ag}$ and $\mathrm{Co}-\mathrm{Cu}$ films [28, 29] reveal non-planar magnetic anisotropy. Detailed and systematic analysis of $(\mathrm{FeCoZr})_{x}\left(\mathrm{CaF}_{2}\right)_{100-x}$ films, sintered on the uncooled substrate (at $T \approx 100-150{ }^{\circ} \mathrm{C}$ ) indicate that high contribution of non-planar magnetic anisotropy is observed within and above percolation threshold $(x \approx$ 46-74). In particular, $M(H)$ curve for $\mathrm{FeCoZr}-\mathrm{CaF}_{2}$ film presented in Fig. 9a shows almost linear dependence at $H<3 \mathrm{kOe}$ and inflection point at $H_{\mathrm{a}} \approx 2-3 \mathrm{kOe}$ when $H$ is applied in parallel direction to the thin film surface.

In addition, the Mössbauer spectroscopy of film with $x \geq 58$ shows magnetically split spectrum (see Fig. 9b) with the ratio of line intensities $h_{3}: h_{2}: h_{1}$ equals to $3: \approx 0.3: 1$. These features of $M(H)$ curves and Mössbauer spectra clearly illustrate that easy magnetization axis lies out of film's plane, that is quite untypical for ion-beam sputtered granular nanocomposite films with random nanoparticles distribution. Angle $\theta$ between normal 

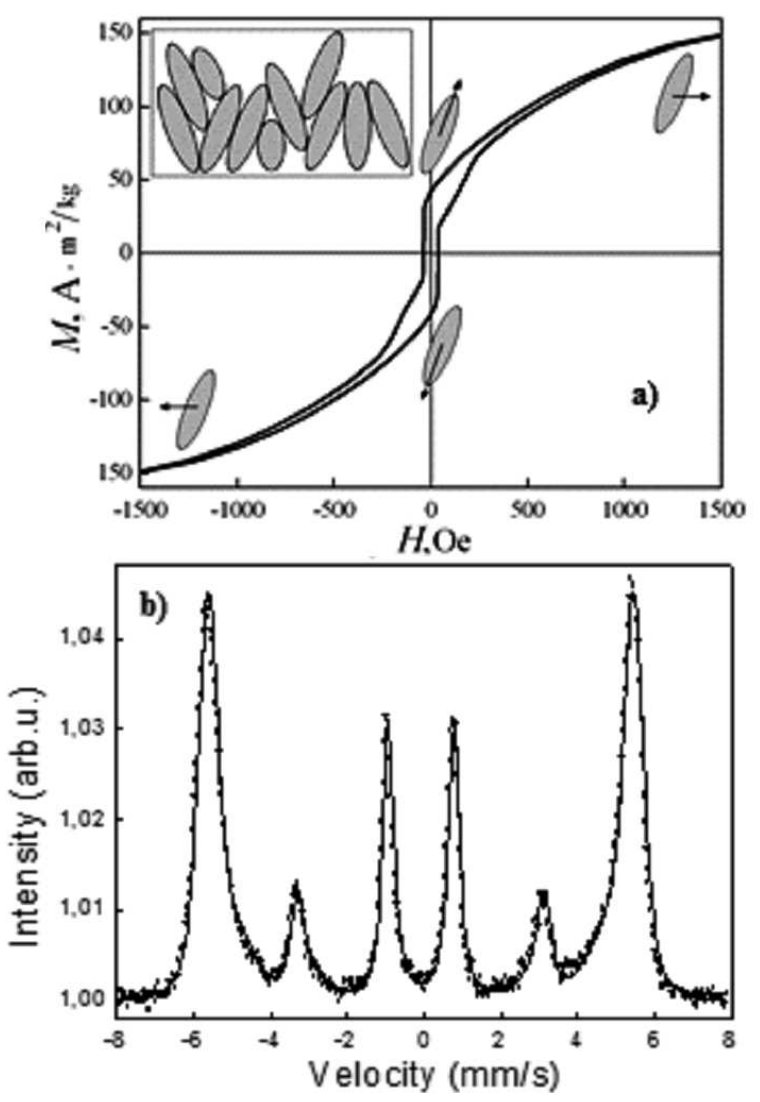

Fig. 9. $\quad M(H)$ curve at $2 \mathrm{~K}(\mathrm{a})$ and ${ }^{57}$ Fe CEMS spectrum at RT (b) of $(\mathrm{FeCoZr})_{67}\left(\mathrm{CaF}_{2}\right)_{33}$ film.

vector to the films surface and the magnetic moment vectors in nanoparticles, estimated basing on experimental results from the relationships $\theta=90^{\circ}-\arccos \left(M_{\mathrm{r}} / M_{\mathrm{s}}\right)$ [23] and $\theta=\arccos ((4-K) /(4+K))^{1 / 2}$ [30], where $M_{\mathrm{r}}$ is remanence and $K=h_{2}: h_{1}$, equals to $\approx 16^{\circ}$ and $20^{\circ}$, correspondingly. It should be mentioned that intrinsic growth-induced magnetic anisotropy is quite unusual phenomenon for granular nanocomposite M-I films.

In the absence of preferable crystallographic orientation of bcc $\alpha-\mathrm{FeCo}(\mathrm{Zr})$ confirmed with XRD, the observed magnetic anisotropy is usually assigned to elongated, ellipsoidal shape of granules [27, 31]. Comparative consideration of structure and magnetic properties of various granular films exhibiting growth-induced magnetic anisotropy reveal dominating role of substrate temperature in promoting this unusual effect with respect to other possible reasons (surface oxidation of nanoparticles, composition of matrix, etc.) $[25-29,31]$.

The effect of substrate temperature on agglomeration of nanoparticles inside insulating matrix could be explained basing on the qualitative model of "self-ordering" agglomeration of atoms and clusters into metallic nanocolumns inside matrix proposed in [32]. This model assumes that growth of column-like nanostructures is associated with concentration of deposited atoms on the surface and the energy of sputtered atoms (or atomic clusters) moving on the substrate surface before atoms impingement. Elevated substrate temperature accelerates surface diffusion of both metallic and insulator atoms (or clusters) and prevents metal and insulator phase separation, so that they can accumulate faster on top of the nucleated underlying grains leading to the growth of columnar structure.

\section{Applications}

Unique combination of magnetic and electric properties of granular films offers some important technological advances to date, particularly making these materials applicable in designing of magnetic sensors and magnetoresistive random access memory devices. In addition, recently observed effect of "negative capacitance" in $(\mathrm{FeCo})_{x}\left(\mathrm{Al}_{2} \mathrm{O}_{3}\right)_{100-x},(\mathrm{FeCo})_{x}\left(\mathrm{CaF}_{2}\right)_{100-x}$ and $(\mathrm{FeCo})_{x}\left(\mathrm{PbZrTiO}_{3}\right)_{100-x}$ granular films with "core-shell" structure of nanoparticles makes them prospective for designing of planar noncoil-like inductances for high-frequency applications compatible with silicon planar technology. Effect of negative capacitance is detected basing on frequency dependences of phase angle shift $\theta(f)$ of voltage against alternating electric current [33]. Typical $\theta(f)$ curves for $\mathrm{FeCo}-\mathrm{Al}_{2} \mathrm{O}_{3}$ films after annealing are shown in Fig. 10a, and demonstrate positive values of the phase angle shift $\theta$ starting from some definite frequency. Negative capacitance effect could be considered as counterpart of the noncoil-like inductive contribution to the film impedance in correspondent equivalent scheme (see Fig. 10b).

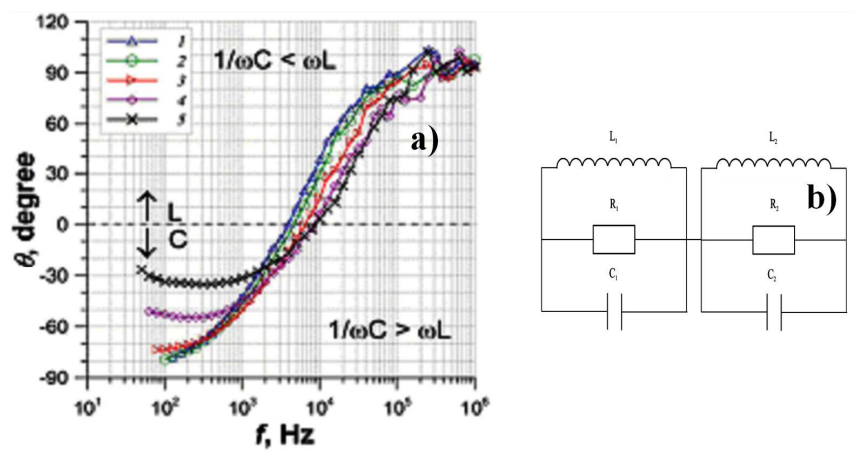

Fig. 10. Typical $\theta(f)$ curves for the as-deposited $\mathrm{FeCo}-\mathrm{Al}_{2} \mathrm{O}_{3}$ films sintered in $\mathrm{Ar}+\mathrm{O}$ atmosphere at measured at $T=153 \mathrm{~K}(1), 178 \mathrm{~K}(2), 218 \mathrm{~K}(3), 258 \mathrm{~K}(4)$, 293 (5) (a) and correspondent equivalent circuit [33] (b).

The value of "effective" inductive contribution to the impedance estimated basing on the correspondent equivalent circuit was found to be as high as $20 \mu \mathrm{H} / \mu \mathrm{m}^{3}$ at $1 \mathrm{MHz}$ that is much higher than in the case of known diodes, $p-n-p$ junctions, metalsemiconductor interfaces (see references in [33]). Interestingly, $(\mathrm{FeCo})_{x}\left(\mathrm{PbZrTiO}_{3}\right)_{100-x}$ films for $x \leq$ 53 at.\% sintered in $\mathrm{Ar}+\mathrm{O}$ atmosphere reveal inductive-like behaviour even without post-synthesis annealing (see Fig. 11a). 


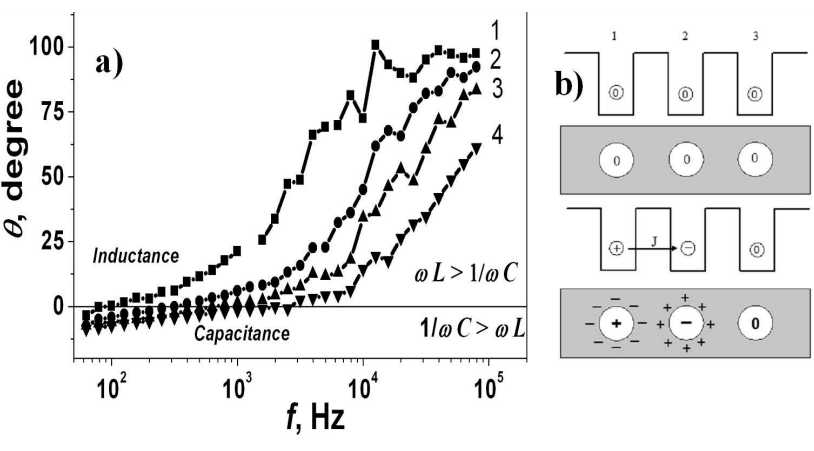

Fig. 11. Typical $\theta(f)$ curves (a) for the as-deposited $(\mathrm{FeCo})_{x}\left(\mathrm{PbZrTiO}_{3}\right)_{100-x}$ films sintered in $\mathrm{Ar}+\mathrm{O}$ atmosphere at measured at $T=243 \mathrm{~K}(1), 263 \mathrm{~K}(2)$, $283 \mathrm{~K}(3), 303 \mathrm{~K}(4)$. Model for AC/DC conductivity inducing the formation of noncoil-like inductive contribution to the impedance in M-I films [34] (b).

Observed "effective" inductive contribution in granular films were interpreted within the frame of the proposed model of $\mathrm{AC} / \mathrm{DC}$ hopping conductance of electrons over selectively oxidized "core-shell" nanoparticles embedded into $\mathrm{Al}_{2} \mathrm{O}_{3}$ and $\mathrm{PbZrTiO}_{3}$ matrixes [34]. Its scheme is shown in Fig. 11b. Positive phase shift angle of the applied voltage against the electric current is related to the formation of dipole - positively and negatively charged nanoparticles - due to the first jump of electron to the neighbouring nanoparticle (potential well). Polarization of dielectric matrix surrounding nanoparticles due to the formed dipole is the origin of the increased electron mean life time in a potential well (nanoparticles) delaying its next jump. Such a time delay resulted in the electric current shift with respect to the applied voltage [33].

\section{Conclusions}

Complementary analysis of structure and phase composition of $\mathrm{M}_{x}-\mathrm{I}_{100-x}$ films with TEM, HRTEM, and XRD proves their granular structure, where basically crystalline bcc FeCo-based nanoparticles are randomly distributed inside amorphous $\left(\mathrm{Al}_{2} \mathrm{O}_{3}, \mathrm{PZT}\right)$ or crystalline $\left(\mathrm{CaF}_{2}\right)$ matrix.

Magnetic and electric properties of granular films demonstrate variety of states that could be effectively tuned by change of metal-to-dielectric fractions ratio. Temperature and field dependences of magnetization curves as well as the Mössbauer spectra clearly reveal the transition from the ensemble of superparamagnetic and weakly coupled bcc FeCo-based nanoparticles in films at $x<40$ towards magnetically percolated net of nanoparticles at $x>50$. The onset of magnetic percolation corresponds to $x_{\mathrm{C}} \approx 50$. Three mentioned concentration ranges are also reflected in the dependences of electrical conductivity on temperature and external magnetic field. Namely, formation of continuous currentconductive cluster (electric percolation around $x_{\mathrm{C}}$ ) becomes evident when crossover from VRH to the metallic mechanism of charge transfer occurs. On the insulating side of the percolation threshold (at $x<x_{\mathrm{C}}$ ) magnetoresistance of $\mathrm{M}_{x}-\mathrm{I}_{100-x}$ films is characterized with spin-dependent tunneling well obeying the Inoue-Maekawa model.

As the consequence of films deposition on substrate at elevated temperature elongated nanogranules are formed demonstrating close to perpendicular magnetic anisotropy.

Synthesis in oxygen-containing atmosphere results in the formation of granules with "metallic core-oxide shell" structure due to selective nanoparticles oxidation when metallic cores constitute bcc FeCo-based solid solution enriched with Co, while oxide shell mainly consists of mixed Fe-rich oxides. Granular films containing "core-shell" nanoparticles are typically characterized with suppressed magnetic and electric percolation which is evidenced by the superparamagnetic state of nanoparticles and VRH carrier transport above the percolation threshold $\left(x_{\mathrm{C}} \approx 50\right)$ as well as by the exchange AFM-FM coupling at the interface between FM core and AFM oxide shell.

Granular films with "core-shell" structure of nanoparticles possess enhanced negative $M R$ effect that could be assigned to the stabilization of the metallic cores sizes within single-domain size as well as to spin accumulation and spin filtering processes.

Effect of negative capacitance is typically observed in the films with "core-shell" structure of nanoparticles. It is related to the formation of dipole between neighbouring nanoparticles and subsequent polarization of dielectric matrix around them. Such a polarization delays next electron's jump and, consequently, results in the delay of electric current with respect to the applied voltage. Granular $\mathrm{M}_{x}-\mathrm{I}_{100-x}$ films with estimated values of effective inductive contribution to the impedance as high as $20 \mu \mathrm{H} / \mu \mathrm{m}^{3}$ at $1 \mathrm{MHz}$ make possible designing of noncoil-like inductances for microelectronics.

\section{Acknowledgments}

Author acknowledges the support from the Belarusian State program "Functional materials", project 1.37, the Polish Ministry of Science and Higher Education, the Serbian Ministry of Education and Science, project OI 171023, EU COST Action MP0903 Nanoalloys, and EU FP7 SILAMPS project 226470. Hasylab/DESY (Hamburg) is also acknowledged for the provision of beam time and financial support within the initiative "ELISA: EU Support of Access to Synchrotrons/FELs in Europe". Special gratitude to Prof. Yu. Kalinin and Dr. hab. A. Sitnikov (Voronezh State Technical University, Russia) for providing the samples, Prof. Cz. Kapusta, Prof. J. Żukrowski, Dr. hab. J. Przewoźnik and Dr. hab. M. Sikora (AGH University of Science and Technology, Faculty of Physics and Applied Computer Science, Department of Solid State Physics, Kraków, Poland) for accomplishment and discussion of XRD, VSM, X-ray 
absorption and Mössbauer spectroscopies results, Prof. M. Milosavljević (VINCA Institute of Nuclear Sciences, Belgrade University, Belgrade, Serbia) for TEM and HRTEM analysis, Prof. P. Żukowski and Dr. T.N. Kołtunowicz (Lublin Polytechnic University, Lublin, Poland) for impedance spectroscopy measurements and elaboration of model of hopping conductance on alternating current, Prof. A. Fedotov (Belarusian State University, Minsk, Belarus) for fruitful discussion on the paper.

\section{References}

[1] L. Liu, B. Li, R. Qin, H. Zhao, X. Ren, Z. Su, Nanotechnology 21, 285701 (2010).

[2] L. Liu, B. Li, J. Ying, X. Wu, H. Zhao, X. Ren, D. Zhu, Z. Su, Nanotechnology 19, 495709 (2008).

[3] E. Thirumal, D. Prabhu, K. Chattopadhyay, V. Ravichandran, Phys. Status Solidi A 207, 2505 (2010).

[4] A. Saad, J. Fedotova, J. Nechaj, E. Szilagyi, M. Marszalek, J. Alloys Comp. 471, 357 (2009).

[5] D.C. Jiles, Acta Mater. 51, 5907 (2003).

[6] S. Ohnuma, H. Fujimori, T. Masumoto, X.Y. Xiong, D.H. Ping, K. Hono, Appl. Phys. Lett. 82, 946 (2003).

[7] Sh. Li, Z. Yuan, J.-G. Duh, J. Phys. D, Appl. Phys. 41, 055004 (2008).

[8] I.V. Zolotukhin, Yu.E. Kalinin, A.T. Ponomarenko, V.G. Shevchenko, A.V. Sitnikov, O.V. Stognei, O. Figovsky, J. Nanostruct. Polym. Nanocomp. 2, 25 (2006).

[9] J. Rodriguez-Carvajal, Physica B 192, 55 (1993).

[10] P. Glatzel, M. Sikora, S.G. Eeckhout, O.V. Safonova, G. Smolentsev, G. Pirngruber, J.A. van Bokhoven, J.D. Grunewaldt, M. Tromp, AIP Conf. Proc. 9th Int. Conf. on Synchrotron Radiation Instrumentation 879, 1731 (2007).

[11] K.V. Klementiev, J. Phys. D, Appl. Phys. 34, 209 (2001).

[12] J. Fedotova, J. Przewoznik, Cz. Kapusta, M. Milosavljević, J. Kasiuk, P. Zukrowski, M. Sikora, A. Maximenko, D. Szepietowska, K.P. Homewood, J. Phys. D 44, 495001 (2011).

[13] T.N. Koltunowicz, P. Zukowski, M. Milosavljević, A.M. Saad, J.V. Kasiuk, J.A. Fedotova, Yu.E. Kalinin, A.V. Sitnikov, A.K. Fedotov, J. Alloys Comp. 586, S353 (2014).

[14] B.D. Hall, D. Sanchet, D. Ugarte, J. Appl. Crystallogr. 33, 1335 (2000).
[15] J. Fedotova, in: Advances in Nanoscale Magnetism, Eds. B. Aktas, F. Mikailov, Springer Proceedings in Physics, Vol. 122, Springer, Berlin 2008, p. 231.

[16] J. Fedotova, J. Kasiuk, J. Przewoznik, Cz. Kapusta, I. Svito, Yu. Kalinin, A. Sitnikov, J. Alloys Comp. 509, 9869 (2011).

[17] Ch.P. Pool, Encyclopedic Dictionary of Condensed Matter Physics, Vol. 1, Elsevier, Oxford (UK) 2004.

[18] L. Néel, C.R. Hebd, Compt. Rend. Acad. Sci. 228, 664 (1949)

[19] D. Stauffer, A. Aharony, Introduction to Percolation Theory, Taylor and Francis Ltd., London 1992, p. 181.

[20] N.F. Mott, J. Non-Cryst. Solids 1, 1 (1968).

[21] B.I. Shklovski, A.I. Efros, Electronic Properties in Doped Semiconductors, Springer Verlag, Berlin, 1984, p. 388.

[22] J. Inoue, S. Maekawa, Phys. Rev. B 53, 927 (1996).

[23] B.D. Cullity, C.D. Graham, Introduction in Magnetic Materials, 2nd ed., Wiley-IEEE Press, New Jersey 2009, p. 359

[24] J. Nogués, J. Sort, V. Langlais, V. Skumryev, S. Suriñach, J.S. Muñoz, M.D. Baró, Phys. Rep. 422, 65 (2005).

[25] Y.J. Chen, T. Suzuki, H. Kanazawa, J. Appl. Phys. 87, 4837 (2000).

[26] Y. Sun, W. Li, P. Dhagat, Ch.R. Sullivan, J. Appl. Phys. 97, 10N301 (2005).

[27] V.M. Kalita, A.A. Timopheev, A.F. Lozenko, S.M. Ryabchenko, A.V. Los, O.V. Stognei, A.V. Sitnikov, J. Appl. Phys. 110, 113918 (2011).

[28] J.Q.C.L. Xiao, C.L.A. Chien, A. Gavrin, J. Appl. Phys. 79, 5309 (1996).

[29] N. Anh Tuan, N.H. Luong, N. Chau, V. Van Hiep, N. Minh Ha, Physica B, Condens. Matter 327, 400 (2003).

[30] F. Bodker, M.F. Hansen, C.B. Koch, K. Lefmann, S. Morup, Phys. Rev. B 61, 6826 (2000).

[31] A.A. Timopheev, I. Bdikin, A.F. Lozenko, O.V. Stognei, A.V. Sitnikov, A.V. Los, N.A. Sobolev, J. Appl. Phys. 111, 123915 (2012).

[32] A.V. Sitnikov, Hab. Thesis, Voronezh State Technical University, Voronezh 2009.

[33] P. Zhukowski, T.N. Kołtunowicz, P. Wegierek, J.A. Fedotova, A.K. Fedotov, A.V. Larkin, Acta Phys. Pol. A 120, 43 (2011).

[34] T.N. Kołtunowicz, J.A. Fedotova, P. Zhukowski, A. Saad, A. Fedotov, J.V. Kasiuk, A.V. Larkin, J. Phys. D, Appl. Phys. 46, 125304 (2013). 